PAPER

\title{
Early cognitive decline in Creutzfeldt-Jakob disease associated with human growth hormone treatment
}

\author{
R J Cordery, M Hall, L Cipolotti, S Al-Sarraj, D G O'Donovan, L Davidson, P Adlard, M N Rossor
}

J Neurol Neurosurg Psychiatry 2003;74:1412-1416

See end of article for authors' affiliations .....................

Correspondence to: Professor Martin Rossor, Dementia Research Group, The National Hospital for Neurology and

Neurosurgery, Queen Square, London WCIN 3BG, UK;

mrossor@dementia.ion. ucl.ac.uk

Received

19 December 2002

In revised form

12 March 2003

Accepted 14 March 2003
Background: Most cases of Creutzfeldt-Jakob disease (CJD) in recipients of human cadaveric growth hormone present with a cerebellar syndrome. Dementia is thought to occur late and as a minor feature of the illness. However, neuropsychology data published on these cases are largely qualitative and anecdotal. The first published case does include a neuropsychological assessment seven months after the onset of a cerebellar syndrome, showing evidence of intellectual decline. Subsequent reports hint that cognitive problems may be present in the initial stages of the illness.

Objective: To assess early cognition in Creutzfeldt-Jakob disease in recipients of pituitary derived human growth hormone.

Methods: Detailed neuropsychology assessment is reported at referral (mean 4.5 months from the onset of symptoms; range 4 to 6 months) in five patients with histologically proven human growth hormone derived CJD.

Results: All cases presented with a cerebellar syndrome and only one had noticed mild memory problems. On formal testing, however, four had demonstrable mild intellectual decline, as measured on the WAIS-R. One case showed selective visual memory impairment and frontal executive dysfunction.

Conclusions: These findings suggest that, although not the presenting feature, mild cognitive decline may be evident in the early stages of CJD associated with human cadaveric growth hormone treatment.
$\mathrm{T}$ he iatrogenic transmission of Creutzfeldt-Jakob disease (CJD) was first recognised in 1974 in a recipient of a corneal graft from a donor who had died of undiagnosed CJD. ${ }^{1}$ Transmission has subsequently been demonstrated following neurosurgery, ${ }^{2}$ stereotactic electroencephalography, ${ }^{3}$ dura mater implants, ${ }^{4}$ and after the administration of human pituitary derived growth hormone and gonadotrophin. ${ }^{56}$ The incubation period and clinical phenotype of the ensuing illnesses vary with the route of inoculation. Where infection is introduced directly into the central nervous system, the incubation period is short (months) and the disease resembles classical sporadic CJD with a progressive dementia syndrome. Inoculation by a peripheral route produces an illness with an incubation period of years (or decades) and a predominantly cerebellar onset. ${ }^{78}$ Epidemiological follow up of all human growth hormone recipients in the USA, published in 1991, confirmed six cases, all presenting with ataxia and with progression to further cerebellar, basal ganglia, and extrapyramidal signs. ${ }^{9}$ It was noted in particular that dementia occurred late and as a minor component of the illness. A review of 34 cases of human growth hormone associated CJD in France confirmed the homogeneity of the clinical phenotype, with the great majority of the patients presenting with ataxia and visual disorders, and dementia occurring around six months from illness onset. ${ }^{10}$ However, the study did highlight the fact that memory disorders may sometimes be present at the onset of the illness (in $25 \%$ of the cases).

Many anecdotal reports suggest that cognitive problems may be an early feature of human growth hormone associated CJD. ${ }^{11-14}$ The first published case, of a 22 year old British woman, is, however, the only report to provide formal neuropsychology data, aquired seven months from the onset of the cerebellar syndrome. ${ }^{515}$ This showed evidence of intellectual decline, with a verbal intelligence quotient (VIQ) of 80.
In order to assess early cognition, we now report detailed assessments at referral in five recipients of human pituitary derived growth hormone with histologically confirmed CJD. The clinical details of cases B, C, D, and E are given with the consent of the patients' family. It was not possible to contact the next of kin of patient A and so details from this case have been further anonymised to retain confidentiality.

\section{CASE REPORTS \\ Case A}

This young, right handed person presented with a three month history of gait ataxia, and subsequently diplopia, dysarthria, incoordination, and poor memory. The patient had received pituitary derived human growth hormone for idiopathic growth hormone deficiency for up to eight years. The patient was a smoker and consumed between 10 and 30 units of alcohol a week. There was no family history of neurological illness. On examination, there were dysarthria, cerebellar ataxia with marked truncal instability, titubation, and tremor of the limbs. There was vertical nystagmus with jerky pursuit movements of the eyes. Myoclonus was seen. The reflexes were symmetrically brisk but with flexor plantar responses. Routine blood investigations and cerebrospinal fluid (CSF) examination were normal, as was an electroencephalogram (EEG). Magnetic resonance imaging (MRI) showed enlargement of the supracerebellar sulci but no signal changes.

The patient died four months after presentation. At necropsy, there was little evidence of cortical atrophy macroscopically, in contrast to the presence of marked atrophy of the cerebellar vermis and superior cerebellar cortex. Microscopically, there was patchy spongiform change throughout the cerebral cortex, with confluent vacuolation in the temporal and occipital lobes. Spongiform change was most prominent in the caudate and putamen, but was also noted in the hippocampus, thalamus, pons, and 
periaqueductal grey matter. There was severe cerebellar involvement with marked loss of granular neurones, severe gliosis, and confluent spongiform change with further gliosis in the white matter. Immunocytochemical staining for prion protein $(\mathrm{PrP})$ was strongly positive in the cerebellar cortex in the granular and molecular layers, with occasional plaques seen. PrP was also seen in the cerebral cortex, basal ganglia, and hippocampus.

\section{Case B}

A 29 year old, right handed man presented with a one year history of impaired balance, six months of twitching in the muscles of the thighs and calves, trembling hands, and weakness in the lower limbs. He had been treated with pituitary derived growth hormone for partial growth hormone deficiency between 1977 and 1981. He was an occasional smoker with minimal alcohol intake. There was no family history of neurological illness. On examination he was very anxious. There was nystagmus on lateral gaze, with jerky pursuit movements of the eyes. Fasciculations were seen in the thighs and calves. There was incoordination of the upper and lower limbs with truncal ataxia. No myoclonus was seen. The reflexes were normal, with flexor plantar responses. Blood investigations, MRI, EEG, and CSF examination were all normal. Sequencing of the open reading frame of the prion protein gene showed no mutations, and the patient was valine homozygous at codon 129 .

As the illness progressed, he became more disinhibited, with hypersexuality and increasing short temperedness. He died seven months from presentation. At necropsy, on macroscopic examination the cerebral hemispheres showed a moderate degree of cortical atrophy. The cerebellar hemispheres and vermis were markedly atrophic. There was widespread spongiform change in the cerebral cortex, most marked in the frontal and temporal lobes. There was also spongiform change in the basal ganglia, thalami, hypothalamus, and brain stem. The cerebellum showed diffuse spongiform change with almost complete depletion of granular neurones, widespread gliosis, and patchy Purkinje cell loss. PrP immunocytochemistry showed a composite pattern of plaque-like lesions in both the cerebral and cerebellar cortices and a finer, granular positivity in and around the areas of spongiform change.

\section{Case C}

A 29 year old man presented with a four month history of ataxia with mild frontal headaches and subsequently tremor, incoordination of the upper limbs, diplopia, dysarthria, and myoclonus. He had received pituitary derived growth hormone from 1977 to 1980 (between the ages of 14 and 16) for idiopathic growth hormone deficiency. There was no family history of neurological illness. On examination he was ataxic. There was a right convergent strabismus with diplopia in all directions. Eye pursuit movements were jerky with hypometric saccades. There was horizontal, oblique, and down-beating nystagmus. He was dysarthric. There was tremor of the outstretched hands and myoclonus. Tone was increased asymmetrically in the lower limbs and incoordination was noted in both the upper and lower limbs. The reflexes were brisk with clonus at the ankle, and plantar responses were flexor. Blood investigations, MRI, EEG, CSF examination, and somatosensory evoked potentials (SSEP) were all normal.

The patient died 11 months from presentation. At necropsy, cerebral and cerebellar atrophy were visible macroscopically, with severe symmetrical atrophy of the caudate, lentiform, and thalamic nuclei. There was widespread severe neuronal loss and reactive gliosis in the cerebral cortex. Spongiform change was only evident where the neuronal loss was less severe-for example, in the hippocampus. Most areas (including the basal ganglia and thalami) had progressed to non-specific status spongiosus. There was almost complete loss of Purkinje and granular cells in the cerebellar vermis.

\section{Case D}

A 32 year old man presented with a three month history of ataxia, intermittent diplopia, and dysarthria. He had a past history of asthma and was given pituitary derived growth hormone from 1977 to 1981 (from the age of 11) for short stature. He was a non-smoker and consumed 30 units of alcohol a week. On examination, he had mild gait ataxia. Pursuit movements of the eyes were jerky in nature. There was no myoclonus and the plantar responses were flexor. Blood investigations were all normal except for a low vitamin B-12 (123 ng/l; normal range 170 to 685). MRI, EEG, visual evoked potentials (VEP), and electromyography (EMG) were all normal. CSF examination showed a mildly raised protein at $0.77 \mathrm{~g} / \mathrm{l}$ (normal range 0.1 to $0.6 \mathrm{~g} / \mathrm{l}$ ), normal glucose, two white cells $/ \mathrm{mm}^{3}, 25$ red cells $/ \mathrm{mm}^{3}$, and no oligoclonal bands. The open reading frame of the prion protein gene was sequenced and no mutations were found. The patient was methionine/valine heterozygous at codon 129.

He died 19 months after presentation. At necropsy, marked atrophy of the cerebellum and reduced volumes of the head of the caudate and the thalamus were seen macroscopically. The frontal and parietal lobes, parahippocampal gyrus, caudate, putamen, thalamus, and cerebellum were most severely affected by vacuolation, reactive gliosis, and proliferative microglial cells. The majority of the plaques seen had a diffuse homogeneous pattern, though a minority had a central core and a pale periphery. The presence of PrP was demonstrated by immunohistochemistry, with the most intense immunoreactivity mirroring the areas with the severest pathological change.

\section{Case E}

A 22 year old woman presented with a six month history of unsteadiness after a flu-like illness. This was followed by hesitant speech and a deterioration in handwriting. She had been treated with pituitary derived growth hormone followed by recombinant growth hormone between the ages of 3 and 17 years (from 1980) for idiopathic growth hormone deficiency. There was no family history of neurological illness. She was a non-smoker and consumed about 7 units of alcohol a week. On examination there was a slowness noted on bedside cognitive testing. Smooth eye pursuit movements were impaired and there was a mild cerebellar dysarthria. She was ataxic, with incoordination of the upper and lower limbs. No myoclonus was detected. The jaw jerk was brisk, and the lower limb reflexes were brisk with flexor plantar responses. Blood investigations were normal except for a mildly raised fasting cholesterol concentrations and a raised serum vitamin E. MRI showed an unrelated small cystic lesion in the left superior frontal gyrus. There was mildly abnormal signal seen in the caudate and lentiform nuclei and thalami. EEG showed minor non-specific abnormalities. Sequencing of the full open reading frame of the prion protein gene showed no mutations, and she was valine homozygous at codon 129. Neuronal markers were raised in the CSF: S100 $2.25 \mathrm{ng} / \mathrm{ml}$ (normal <0.38), NSE $67 \mathrm{ng} / \mathrm{ml}$ (normal $<20$ ), and positive protein 14-3-3.

As the illness progressed the patient's behaviour became increasingly child-like and she died five months from presentation. At necropsy, there was a moderate degree of atrophy of the cerebellum macroscopically. There was extensive spongiform change and gliosis affecting the frontal cortex (and the Sylvian fissure region), hippocampus, 
caudate, putamen, and thalamus. In the cerebellum there was marked loss of neurones from the granular layer, proliferation of Bergman glia, and also isomorphic gliosis and spongiform change in the molecular cortex. There was relative preservation of Purkinje cells. Immunohistochemistry for prion protein showed deposits following the distribution of the spongiform change. The deposits were mostly in a synaptic pattern in the cerebrum, though there were some dense aggregates in the globus pallidus and thalamus. There were extensive clumped deposits in the granular cell layer of the cerebellum, and a synaptic pattern in the molecular cortex.

\section{Neuropsychology}

The results of a comprehensive neuropsychology assessment were available for all five patients (at 4, 12, 4, 3, and 6 months after the first symptoms, and after approximately $57 \%, 63 \%, 27 \%, 14 \%$, and $55 \%$ of the total illness duration, respectively). This included standardised measures of general intellectual functions, memory, language, and perceptual, spatial, and frontal executive skills.

\section{General intellectual functions}

General intellectual functions for all five patients were assessed by administering the shortened version of the Wechsler adult intelligence scale-revised (WAIS-R), ${ }^{16}{ }^{17}$ consisting of four verbal subtests and three non-verbal subtests. Verbal and performance IQs were obtained by prorating verbal and performance subtest scores. The national adult reading test (NART) was administered to obtain a reading IQ equivalent score, giving an estimate of the individual's optimum premorbid level. ${ }^{18} 19$ The discrepancy between the NART IQ scores and WAIS-R IQ scores provides an estimate of the severity of intellectual deterioration. The WAIS-R IQ scores and NART full scale IQ equivalent are given for each patient in table 1.

Patient A performed in the lower end of the average range on the verbal and the non-verbal scales of the WAIS-R. The performance was also in the lower end of the average range on the Raven's advanced matrices, a test of non-verbal abstract reasoning $(7 / 12) .^{20}$ The patient's estimated optimal premorbid level of functioning was in the average range. Scores on tests of general intelligence, therefore, indicated a significant degree of intellectual deterioration, particularly affecting non-verbal skills.

The performance of patient B was within the average range on both scales of the WAIS-R but was nevertheless below his premorbid optimal level of functioning.

Patient $\mathrm{C}$ performed in the average range on the verbal scale and in the low average on the non-verbal scale. His reading IQ equivalent on the NART was in the upper end of the average range, thus indicating a significant deterioration of his general intellectual skills, particularly his non-verbal skills.

Patient D performed in the average range on both scales of the WAIS-R. His estimated optimal premorbid level was in the high average range; thus the results indicated a significant degree of intellectual deterioration.

Patient E attained an average VIQ and a low average PIQ. On the advanced matrices test her performance was in the low average range (6/12). Her premorbid level of functioning, as estimated by the NART, was in the high average range. Thus there was a significant degree of intellectual decline on tests of general intelligence with a verbal component, and a moderate degree of decline on tests with a non-verbal component.

To summarise, there was evidence of significant intellectual deterioration, particularly affecting non-verbal skills in four of the five patients.

\section{Memory skills}

The recognition memory test (RMT) was administered to all the patients to obtain a measure of verbal and visual memory skills. ${ }^{21}$ In addition, one patient (E) was given one verbal and one visual memory test from the Camden memory test. ${ }^{22}$ The raw scores and centile scores for the RMT are given in table 2 .

Four of the five patients performed satisfactorily on the RMT. However, patient E had a selective weakness of visual memory with a defective score in the visual version of the RMT (32/50 faces) and a low average score on the topographical memory test (23/30). By contrast, she obtained superior scores on the verbal version of the RMT (words 49/ 50). Her performance was also superior on the paired associates learning test ( $\mathrm{T} 124 / 24 ; \mathrm{T} 2$ 24/24). Overall, memory function was within normal limits in this series of patients except for patient E, who presented with a selective visual memory impairment.

\section{Other cognitive skills (naming, visual perceptual,} visuospatial, and frontal executive skills)

The graded naming test was administered to obtain a measure of nominal skills. ${ }^{23}$ Four of the subtests of visual perceptual and space perception battery ${ }^{24}$ were administered to obtain a measure of visual perceptual (object decision and fragmented letters) and visuospatial skills (cube analysis and number location). Further, the cognitive estimates test, ${ }^{25}$ the Weigl test, ${ }^{26}$ and the verbal fluency test (words beginning with the letter $S)^{27}$ were administered to assess frontal executive functions. All of the patients had normal naming, visual perceptual, and visuo-spatial function (table 3 ).

\begin{tabular}{llllll}
\hline \multicolumn{5}{l}{ Table 1 } & WAIS-R IQ scores and NART full scale IQ equivalent for each of the patients \\
\hline Subject & A & B & C & D & E \\
\hline Timing of assessment (months from & 4 & 12 & 4 & $3-4$ & 6 \\
illness onset) & & & & & 94 \\
WAIS-R VIQ & 91 & 92 & 99 & 91 & 83 \\
WAIS-R PIQ & 85 & 93 & 87 & 92 & 88 \\
WAIS-R FIQ & 87 & 92 & 93 & 91 & 113 \\
NART FIQ & 100 & 100 & 107 & 110 & \\
Scaled scores & & & & 9 & 6 \\
Digit span & 8 & 7 & 13 & 9 & 11 \\
Vocabulary & 8 & 10 & 10 & 7 & 9 \\
Arithmetic & 10 & 12 & 7 & 10 & 10 \\
Similarities & 9 & 8 & 11 & 9 & 8 \\
Picture completion & 8 & 11 & 9 & 7 & 8 \\
Picture arrangement & 7 & 8 & 8 & 10 & 8 \\
Block design & 9 & 8 & 7 & & \\
\hline
\end{tabular}


Table 2 Raw and centile scores for the recognition memory test

\begin{tabular}{llllll}
\hline Subject & A & B & C & D & E \\
\hline $\begin{array}{l}\text { Timing of assessment (months from illness onset) } \\
\begin{array}{l}\text { Words } \\
\text { Scores }\end{array}\end{array}$ & 4 & 12 & 4 & $3-4$ & 6 \\
(Centiles) & $46 / 50$ & $43 / 50$ & $49 / 50$ & $49 / 50$ & $49 / 50$ \\
Faces & $(25$ to 50$)$ & $(10$ to 25$)$ & $(>90)$ & $(>90)$ & $(>95)$ \\
Scores & $43 / 50$ & $44 / 50$ & $46 / 50$ & $48 / 50$ & $32 / 50$ \\
(Centiles) & $(50)$ & $(50$ to 75$)$ & $(75)$ & $(90)$ & $(<5)$ \\
\hline
\end{tabular}

Finally, all except one patient (E) performed within normal limits on tests sensitive to frontal lobe dysfunction. Patient $\mathrm{E}$ passed the Weigl sorting test and the Wisconsin card sorting test. ${ }^{28}$ However, her phonemic fluency for words beginning with the letter $\mathrm{S}$ was markedly reduced. Her performance was similarly impaired for two further phonemic categories $(\mathrm{F}=6, \mathrm{~A}=6)$.

There was evidence of some cognitive slowing in patients A and D. On a test of speed and attention (reciting the months of the year backwards), the performance of patient A was noted to be slow and inefficient. Patient $\mathrm{D}$ was also noted to perform slowly during assessments.

In summary, all the other patients performed within normal limits on tests of naming, visual perceptual, and visuospatial function. Evidence of visual memory impairment and frontal executive dysfunction was found in patient $\mathrm{E}$.

\section{DISCUSSION}

There are still small numbers of cases of CJD occurring in recipients of cadaveric pituitary derived human growth hormone. These represent disease onsets after very long incubations periods, with inoculation predating the introduction of recombinant growth hormone in mid-1985. The illness is rare and difficult to diagnose in the early stages, and it is hard to exclude human pituitary derived growth hormone recipients who are well but concerned. Some cases of sporadic CJD may present with ataxia. Following one classification, these may be VV2 or MV2 cases according to the polymorphism for methionine and valine at codon 129 and the physicochemical properties of the abnormal prion protein. ${ }^{29}$ Approximately one third of MV2 cases present under the age of 40 years and they often have an illness duration of over one year. These cases may have cognitive impairment from an early stage. Sporadic CJD and human growth hormone associated CJD may therefore be difficult to distinguish clinically in the early stages if a detailed past medical history is not taken.
Neuropsychology data in reported cases of human growth hormone associated CJD are largely qualitative but suggest that cognitive problems may feature early in the illness. Koch et al described a 20 year old man who presented with a three month history of an unsteady gait, followed shortly by dysarthria. ${ }^{11}$ It is recorded that on admission "though he was orientated and responsive, his mentation was impaired."

Croxson et al described a 31 year old woman from New Zealand who presented with clumsiness and an unsteady gait. ${ }^{12}$ On examination three months after her initial symptoms it was recorded that "she was orientated in time and place but short term memory was impaired." Similarly, Marzewski et al described a 37 year old man who presented with a one month of progressive imbalance. ${ }^{13}$ Neuropsychology testing revealed "borderline cognitive impairment with possibly slightly reduced learning efficiency." The authors, however, remarked on the relative preservation of mental function at presentation.

Masson et al reported two French cases who showed no evidence of intellectual decline at presentation (two and three months from the onset of symptoms) but who rapidly developed obvious cognitive problems (five and four months from disease onset, respectively). ${ }^{14}$

We have reviewed five cases with detailed neuropsychology data. All presented with a cerebellar syndrome and only one had noticed mild memory problems. The other four cases and their relatives all denied noticing any cognitive problems. On formal testing, however, four had demonstrable mild but significant intellectual decline at this early stage in their illness (mean 4.4 months from the onset of symptoms, range 4 to 6 months). One case showed a selective visual memory impairment and frontal executive dysfunction. Though recorded subjectively, cognitive impairment seemed to remain a minor feature in cases $\mathrm{B}, \mathrm{D}$, and $\mathrm{E}$, who remained in contact with our counselling services.

All of the cases had widespread disease affecting the cerebrum and cerebellum at necropsy. Spongiform change,

Table 3 Scores for tests of naming, visual perceptual, visuospatial, and frontal executive skills

\begin{tabular}{|c|c|c|c|c|c|}
\hline Subject & A & B & $\mathrm{C}$ & D & $\mathbf{E}$ \\
\hline $\begin{array}{l}\text { Timing of assessment (months from illness onset) } \\
\text { Naming skills }\end{array}$ & 4 & 12 & 4 & $3-4$ & 6 \\
\hline GNT (score) & $20 / 30$ & $22 / 30$ & $21 / 30$ & $22 / 30$ & $23 / 30$ \\
\hline (Centile) & $(25$ to 50$)$ & $(50$ to 75$)$ & $(50)$ & $(50$ to 75$)$ & $(50$ to 75$)$ \\
\hline \multicolumn{6}{|l|}{ Visual perceptual skills (VOSP) } \\
\hline Fragmented letters (score) & NT & $19 / 20$ & $20 / 20$ & $20 / 20$ & NT \\
\hline Object decision (score) & $19 / 20$ & $18 / 20$ & $20 / 20$ & $19 / 20$ & $24 / 30$ \\
\hline \multicolumn{6}{|l|}{ Visuospatial skills } \\
\hline Cube analysis (score) & $10 / 10$ & $10 / 10$ & $10 / 10$ & NT & $9 / 10$ \\
\hline Number location (score) & NT & NT & NT & $9 / 10$ & NT \\
\hline \multicolumn{6}{|l|}{ Frontal executive skills } \\
\hline Cognitive estimates errors & 1 (pass) & NT & 1 (pass) & 0 (pass) & 4 (pass) \\
\hline Weigl (No of solutions) & 2 & 2 & NT & 2 & 2 \\
\hline Fluency (words beginning with S) & 19 & 33 & 22 & 22 & 3 \\
\hline
\end{tabular}


neuronal loss, and gliosis-features characteristic of Creutzfeldt-Jakob disease-were particularly severe in the cerebellum but also marked in the frontal lobes. Disturbances of executive function and visuospatial problems, as seen in patient E, are usually associated with lesions in the prefrontal and parietal cortices, respectively. However, these features and the mild decline in intellectual performance seen in cases A, C, D, and E could also be consistent with a cerebellar cognitive affective syndrome. ${ }^{30}$ This term describes a pattern of neuropsychological and behavioural abnormalities in patients with acute cerebellar disease, thought to arise from disruption of corticopontine/pontocerebellar and cerebellothalamic/thalamocortical pathways. The syndrome is characterised by personality change (ranging from disinhibited behaviour to a blunting of affect); impairment of executive functions, perseveration, and inattention; visual-spatial impairment; and abnormalities of language production and anomia. The syndrome has been associated in particular with posterior lobe and vermal lesions. The earliest reported symptoms in all of our cases were associated with cerebellar dysfunction. It could be postulated that the cognitive decline noted at this early stage may be caused by disease affecting connections between the parietal, temporal, prefrontal, and limbic cortices and the cerebellum, resulting in a general reduction in intellectual functioning, as seen in the cerebellar cognitive affective syndrome.

We suggest that cognitive impairment is present in the early stages of human growth hormone associated CJD but it is, and often remains, a minor component of the illness.

\section{ACKNOWLEDGEMENTS}

We would like to acknowledge Professor James Ironside, Department of Neuropathology, The National Creutzfeldt-Jakob Disease Surveillance Unit, Western General Hospital, Edinburgh, Dr T H Moss, Department of Neuropathology, Frenchay Hospital, Bristol, and Dr J Anderson, Department of Neuropathology, Addenbrooke's Hospital, Cambridge, UK, for access to and review of the neuropathological data.

\section{Authors' affiliations}

R J Cordery, M N Rossor, Dementia Research Group, Institute of Neurology, University College London and Division of Neuroscience and Psychological Medicine, Faculty of Medicine, Imperial College of Science, Technology and Medicine, London WCI, UK

M Hall, L Cipolotti, Department of Neuropsychology, The National Hospital for Neurology and Neurosurgery, London WC1, UK S Al-Sarraj, Department of Neuropathology, Institute of Psychiatry, King's College London, London SE5, UK

D G O'Donovan, Department of Neuropathology, Addenbrooke's Hospital, Cambridge, UK

L Davidson, P Adlard, Biochemistry, Endocrine and Metabolism Unit, Institute of Child Health, London WCl, UK

Competing interests: none declared

\section{REFERENCES}

1 Duffy P, Wolf J, Collins G, et al. Possible person-to-person transmission of Creutzfeldt-Jakob disease. N Engl J Med 1974;290:692-3.
2 Will RG, Matthews WB. Evidence for case-to-case transmission of CreutzfeldtJakob disease. J Neurol Neurosurg Psychiatry 1982;45:235-8.

3 Bernoulli C, Siegfried J, Baumgartner G. Danger of accidental person-toperson transmission of Creutzfeldt-Jakob disease by surgery [letter]. Lancet 1977;i:478-9.

4 Thadani V, Penar PL, Partington J, et al. Creutzfeldt-Jakob disease probably acquired from a cadaveric dura mater graft. J Neurosurg 1988;69:766-9.

5 Powell-Jackson J, Kennedy P, Whitcombe EM, et al. Creutzfeldt-Jakob disease and administration of human growth hormone. Lancet 1985;ii:244-6.

6 Cochius JI, Burns RJ, Blumberg PC, et al. Creutzfeldt-Jakob disease in a recipient of human pituitary-derived gonadotrophin. Aust NZ J Med 1990;20:592-3.

7 Brown P, Preece MA, Will RG. "Friendly fire" in medicine: hormones, homografts, and Creutzfeldt-Jakob disease. Lancet 1992;340:24-7.

8 Brown P, Preece M, Brandel J-P, et al. latrogenic Creutzfeldt-Jakob disease at the millennium. Neurology 2000;55:1075-81.

9 Fradkin JE, Schonberger LB, Mills JL, et al. Creutzfeldt-Jakob disease in pituitary growth hormone recipients in the United States. JAMA $1991 ; 265: 880-4$

10 Billette de Villemeur T, Deslys J-P, Pradel A, et al. Creutzfeldt-Jakob disease from contaminated growth hormone extracts in France. Neurology 1996;47:690-5.

11 Koch TK, Berg BO, De Armond SJ, et al. Creutzfeldt-Jakob disease in a young adult with idiopathic hypopituitarism. Possible relation to the administration of cadaveric human growth hormone. N Engl J Med 1985;313:731-3.

12 Croxson M, Brown P, Synek B, et al. A new case of Creutzfeldt-Jakob disease associated with human growth therapy in New Zealand. Neurology 1988;38:1128-30.

13 Marzewski DJ, Towfighi J, Harrington MG, et al. Creutzfeldt-Jakob disease following pituitary-derived human growth hormone therapy: a new American case. Neurology 1988;38:1131-4.

14 Masson C, Delalande I, Deslys JP, et al. Creutzfeldt-Jakob disease after pituitary-derived human growth hormone therapy: two cases with valine 129 homozygous genotype. Neurology 1994;44:179-80.

15 Weller RO, Steart PV, Powell-Jackson JD. Pathology of Creutzfeldt-Jakob disease associated with pituitary-derived human growth hormone administration. Neuropathol Appl Neurobiol 1986;12:117-29.

16 Wechsler D. Manual for the WAIS, revised. New York: Psychological Corporation, 1981

17 Warrington EK, James M, Maciejewski C. The WAIS as a lateralizing and localizing diagnostic instrument: a study of 656 patients with unilateral cerebral lesions. Neuropsychologia 1986;24:1233-40.

18 Nelson HE. National adult reading test (NART): for the assessment of premorbid intelligence in patients with dementia. Windsor: NFER-Nelson, 1982.

19 Nelson HE, Willison JR. Re-standardisation of the NART against the WAIS-R. Windsor: Nelson, 1992.

20 Raven JC, Court JH, Raven J. The coloured progressive matrices. Oxford: Oxford Psychologists' Press, 1990.

21 Warrington EK. The recognition memory test. Windsor: NFER-Nelson, 1984.

22 Warrington EK. The Camden memory tests manual. Hove: Psychology Press, Hove, 1996.

23 McKenna P, Warrington EK. The graded naming test. Windsor: NFERNelson, 1983.

24 Warrington EK, James M. The visual perceptual and space perception battery. Bury St Edmunds: Thames Valley Test Co, 1991.

25 Shallice T, Evans ME. The involvement of the frontal lobes in cognitive estimation. Cortex 1978;14:294-303.

26 Weigl E. Zur psychologie sogenannter Abstraktionsprogesse. Z Psychol 1927; 103:2-45, [Translated by Rioch $M$, and reprinted On the psychology of so called processes of abstraction J Abnorm Soc Psychol 1948;36:3-33].

27 Spreen O, Strauss E. A compendium of neuropsychological tests: administration, norms and commentary. Oxford: Oxford University Press, 1998.

28 Nelson HE. A modified card sorting task sensitive to frontal lobe defects. Cortex 1976;12:313-24.

29 Parchi P, Giese A, Capellari S, et al. Classification of sporadic CreutzfeldtJakob disease based on molecular and phenotypic analysis of 300 subjects. Ann Neurol 1999;46:224-33.

30 Schmahmann JD, Sherman JC. The cerebellar cognitive affective syndrome. Brain 1998;121:561-79. 